\title{
L’étude des unions en démographie : des catégories aux processus
}

STUDYING MARRIAGE, COHABITATION, AND OTHER

NON-EPHEMERAL SEXUAL UNIONS: CROSS-SECTIONAL CATEGORIES VERSUS MULTIDIMENSIONAL CONTINUA AND PROCESS

\section{ESTUDIO DE LAS UNIONES (MATRIMONIO, COHABITACION) EN DEMOGRAFIA : CATEGORIAS Y PROCESOS}

\section{Thomas K. Burch et Danièle Bélanger}

Volume 28, numéro 1-2, printemps-automne 1999

L'union libre

URI : https://id.erudit.org/iderudit/010258ar

DOI : https://doi.org/10.7202/010258ar

Aller au sommaire du numéro

Éditeur(s)

Association des démographes du Québec

ISSN

0380-1721 (imprimé)

1705-1495 (numérique)

Découvrir la revue

Citer cet article

Burch, T. K. \& Bélanger, D. (1999). L'étude des unions en démographie : des catégories aux processus. Cahiers québécois de démographie, 28(1-2), 23-52. https://doi.org/10.7202/010258ar

\section{Résumé de l'article}

Les auteurs soutiennent que les outils dont la démographie classique se sert pour étudier le mariage et la famille ne peuvent appréhender adéquatement les formes actuelles d'union, qui sont diverses et mouvantes. En mettant l'accent sur les processus plutôt que sur les états et sur l'appartenance à des catégories, on rendrait mieux justice au fait que des « états " comme le mariage et la cohabitation s'insèrent dans des parcours de vie dont la succession ne va pas sans chevauchements. La saisie des processus suppose l'examen des prises de décisions des individus et des couples qui sous-tendent leurs mouvements entre différentes catégories. L'étude et la définition des unions à caractère sexuel bénéficieraient d'une plus grande utilisation des méthodes quantitatives modernes, analyse factorielle, analyse de groupement et logique des ensembles flous notamment. Les méthodes qualitatives sont particulièrement adaptées à l'étude des processus et des significations. Les plus grands progrès viendront d'une combinaison des approches quantitatives et qualitatives. 
Cahiers québécois de démographie

Vol. 28, no 1-2, printemps-automne 1999, p. 23-52.

\title{
L'étude des unions en démographie : des catégories aux processus
}

\author{
Thomas K. BURCH et Danièle BÉLANGER *
}

Rares sont, près de nous comme ailleurs, les sociétés où la loi et la coutume ont approuvé sans réserve les relations stables à caractère sexuel, sauf dans le cadre strict et impératif du mariage légal. Ainsi trouve-t-on souvent, dans les recensements et les répertoires de données de l'Amérique latine du XIXe siècle et du début du XXe siècle, des lieux où la majoritê des naissances portent la mention "illégitime ", les parents n'étant pas devenus mari et femme en vertu d'une cérémonie religieuse officielle, bien que leur petite communauté (généralement pauvre) les reconnût comme tels, et même s'ils allaient passer leur vie ensemble. Durant des siècles, les enfants "illégitimes " - nés hors d'un mariage civil ou béni dans une église - ont systématiquement été infériorisés par la loi et la religion.

Cette conception légaliste perdure et entrave aujourd'hui nos efforts pour ajuster nos lois, notre organisation sociale et nos savoirs aux nouvelles formes d'union hors-mariage. Elle a aussi, par le passé, empêché les études comparatives, dans la mesure où nous avons voulu faire entrer dans nos catégories occidentales des systèmes sociaux qui y sont irréductibles.

Elle a également marqué les recensements et la démographie de son empreinte, donnant lieu à la classification bien connue des populations en fonction des statuts de célibataire (personne n'ayant jamais été mariée légalement), marié(e), divorcé(e) ou veuf (veuve), catégories auxquelles on ajoute parfois celle de séparé(e). L'avènement des formes modernes de cohabitation brouille cette approche. Au Canada, il n'existe

Population Studies Centre, University of Western Ontario, London, Ontario,Canada.burch@julian.uwo.ca dbelang@julian.uwo.ca 
même pas de terme commun aux deux langues officielles pour désigner la cohabitation d'un couple non marié; or si, en anglais, le terme common-law marriage sous-entend l'existence d'un cadre légal, le français union libre en connoterait plutôt l'absence. On comprend qu'il ait été difficile à Statistique Canada de décider si la cohabitation relève de la rubrique "état matrimonial " ou n'est qu'une " relation " au sein d'un ménage.

Lorsque la cohabitation est apparue, certains ont voulu y voir une forme de mariage, une période d'essai précédant les formalités, ou une solution de rechange, voire une protestation à l'égard du mariage légal : une forme plus libre de mariage. Dans un article marquant, Rindfuss et Vanden Heuvel (1990) ont voulu trancher la question en assimilant les cohabitants aux célibataires, faisant valoir que, par leur comportement, ils sont plus proches de ces derniers que des personnes mariées. Carmichael, après une revue complète de la littérature (1995), conclut que l'on peut tout au plus considérer la cohabitation hors mariage comme un stade tardif des fréquentations. Villeneuve-Gokalp (1990) distingue pour sa part divers types de cohabitation, plus ou moins proches du mariage ou du célibat.

Le point de vue que nous soutiendrons dans cet article est que ces unions, parce qu'elles sont trop diverses et trop mouvantes, échappent au filet de la démographie classique, où prévaut la tendance à ranger les unions individuelles dans une seule des catégories correspondant à un nombre limité d'états ou statuts discrets, eux-mêmes définis en fonction d'un nombre réduit de critères simples, concrets, "objectifs ", telles la légalité, la durée ou la corésidence au sein d'un ménage de recensement. Même les approches plus dynamiques, comme celles qui exploitent les modèles multi-états, se bornent à étudier les mouvements des individus au sein des catégories traditionnelles.

Il serait plus fécond de reconnaître que la formation des couples est un processus multidimensionnel. En situant adéquatement les unions existantes par rapport à des dimensions prises isolément, on peut certes arriver à les ranger dans des catégories bien délimitées; mais il n'est pas du tout sûr qu'il s'agira des catégories d'état matrimonial reconnues. Il n'y a pas lieu de s'en êtonner, car les mariages légaux eux-mêmes ont souvent présenté des différences sur des aspects importants. Dans l'histoire occidentale, par exemple, le mariage morganatique, bien qu'il unît en toute légalité un homme de haut rang à une femme de condition sociale inférieure, niait à celle-ci les 
droits habituels d'une épouse sur l'héritage de son mari. L'indissolubilité du mariage a été proclamée et observée à plus d'une époque et sous bien des latitudes. Ailleurs et en d'autres temps, il a été facile d'obtenir le divorce (la rupture officielle d'un mariage légal). Certaines sociétés ont imposé la monogamie; d'autres ont vu un idéal de vie dans la possibilité d'avoir de multiples partenaires et, souvent, l'ont réalisé. Le respect de quelques exigences minimales étant assuré par un contrat de mariage valide, religions et gouvernements se sont volontiers désintéressés des questions de motivations, de sens et de sentiments liées à la mise en union. Et la catégorie de recensement marié a jeté le voile sur l'hétérogénéité réelle des comportements.

La liste complète des dimensions dont il conviendrait de tenir compte pour étudier les formes d'union reste à faire, mais elle devrait comprendre au minimum les éléments suivants : les intentions des parties en ce qui concerne la durée de leur union, la procréation, le mariage, etc.; le sexe des partenaires; le sérieux de leurs sentiments; leur statut légal; l'existence d'un rite inaugural et sa nature; le statut accordé à l'union au sein de la communauté; la fidélité; le mode de dissolution de l'union. $\mathrm{Au}$ moins deux de ces éléments - les intentions des parties et la perception de leur union par le milieu - soulèvent des questions de sens et posent un problème épineux, car les partenaires n'entretiennent pas forcément les mêmes significations l'un que l'autre ou que l'Église, l'État, la communauté, la famille ou la statistique officielle.

De cette approche multidimensionnelle pourrait résulter un nouvel ensemble de catégories non ethnocentriques et véritablement applicables à un éventail de sociétés plus étendu dans le temps et dans l'espace. Nous en tirerions à tout le moins une conscience plus vive du fait que les modes de formation des couples de certaines sociétés, différant qualitativement des nôtres, nécessitent d'autres catégorisations. Il s'agit en somme d'exploiter des preuves empiriques plutôt que d'imposer des postulats et des classifications a priori.

Un autre élément de la démarche proposée ici est la notion de processus, dont la prise en compte permet de situer les "états" que sont le mariage et la cohabitation au sein des nombreux états susceptibles de jalonner les parcours imbriqués du cycle de vie. La signification d'une situation de cohabitation dépend, dans un sens, des états sur lesquels elle débouche. La saisie de ce processus suppose en outre l'examen 
des prises de décisions des individus et des couples, sousjacentes à leur cheminement à travers les statuts de la panoplie démographique. Ces processus de décision sont généralement mal mesurés, voire carrément mis de côté.

Il nous faut donc renouveler nos démarches de recherche. Deux avenues sont à retenir :

- premièrement, une approche quantitative comportant 1'utilisation plus systematique des méthodes de réduction des dimensions telles que l'analyse factorielle et l'analyse de groupement, négligées jusqu'ici par la démographie nordaméricaine, de même que des nouvelles méthodes d'analyse des parcours biographiques;

- deuxièmement, un recours accru aux méthodes qualitatives. Les progrès les plus décisifs dans la compréhension des formes actuelles d'union seront apportés par l'association de ces approches aux méthodes quantitatives (et aux analyses multivariées plus traditionnelles), sinon dans le cadre des mêmes projets de recherche, du moins à l'intérieur de synthèses théoriques susceptibles de tirer parti des remarquables acquis des deux démarches.

\section{DÉMOGRAPHIE ET FORMES D'UNION : BILAN ET PROSPECTIVE}

Il nous arrive d'oublier que la démographie, comme toute discipline scientifique, décide de ses objets de recherche et de ses méthodes d'analyse en opérant des choix parmi un ensemble de possibles. C'est ainsi qu'une science prend forme, ou se déforme, dans la mesure où ces décisions diminuent sa capacité de cerner les réalités qu'elle entend éclairer. S'ils font sa force et lui donnent sa cohérence, ses choix fixent aussi ses limites. Ainsi la démographie a-t-elle pris par le passé certaines orientations déterminantes :

- elle s'est tournée vers les études empiriques, au détriment des synthèses théoriques;

- elle s'est appuyêe sur les recensements et sur de vastes enquêtes sur échantillon, généralement réalisés par les services de la statistique de l'État ou commandités par ce dernier;

- elle a travaillé sur des variables dites "objectives ", en particulier sur des événements discrets directement observables et faciles à situer sur le calendrier; 
- elle a préféré l'étude des événements à celle des processus qui les engendrent;

- elle a tendu à négliger les comportements qui, entachés dle tabous, échappent en partie aux enquêtes officielles et aux instruments de recherche classiques;

- elle a constamment privilégié les approches statiques (l'analyse transversale) et les modèles d'équilibre (analyse des populations stationnaires, théorie micro-économique des comportements, modèles de régression atemporels); mais les méthodes actuelles d'analyse des données biographiques, qui prennent en compte une multiplicité d'états, phénomènes et caractères variables dans le temps, constituent un net progrès, même par rapport à l'analyse longitudinale classique;

- elle a fait de l'individu, plutôt que de la famille ou du milieu, le pivot de ses analyses;

- elle a concentré son attention sur les comportements dictés par des décisions rationnelles et informées, maximisant l'utilité à long terme des agents;

- elle a recouru à des typologies ou classifications relativement simples, axées sur les catégories de recensement traditionnelles.

Ces choix limitent notre capacité d'étudier les nouveaux types de comportements qui sont apparus en Occident durant la révolution sexuelle des années 1960 et continuent de se répandre à travers le monde. La recherche démographique, nous l'avons dit, a tenté d'insérer la cohabitation dans la classification usuelle axée sur l'état matrimonial et basée sur le statut légal. Les cohabitants sont-ils avant tout des "célibataires" arrivés à un "stade tardif des fréquentations"? La cohabitation est-elle une forme de mariage ? Ou s'agit-il d'une catégorie distincte qui devrait s'ajouter à celles de célibataire, marié, séparé, divorcé, veuf? Faudra-t-il inventer des catégories pour rendre compte des ruptures entre cohabitants?

Les données exploitées par les démographes continuent de provenir principalement des recensements et de vastes enquêtes sur échantillon - y compris les enquêtes longitudinales qui recueillent des données biographiques - reposant sur des questionnaires normalisés. La récolte est composée surtout d'événements discrets directement observables (tel le début de la cohabitation) et d'indicateurs "objectifs " basés sur des faits d'expérience (par exemple le moment où est survenue 
la séparation physique du couple). Les comportements tabous sont négligés, car les enquêtes officielles peuvent difficilement précéder l'opinion publique de trop loin, ou solliciter des informations sur des pratiques déviantes ${ }^{1}$. Malgré certains progrès, notamment en ce qui concerne l'union libre, les données sur des expériences comme l'avortement et les relations homosexuelles continuent d'échapper aux recensements ou enquêtes les plus soigneusement conçus et réalisés.

Les méthodes d'analyse multidimensionnelle usitées en démographie, en particulier les modèles à risques proportionnels ou les modèles multi-états, très en faveur en ce moment, s'appuient également sur les catégories classiques de ces recensements et enquêtes.

La tendance compréhensible à définir le concept de cohabitation eu égard au mariage peut expliquer le sursaut causé par la découverte que les mariages précédés de cohabitation présentent des taux de divorce supérieurs et non inférieurs à ceux du mariage direct, contrairement à ce qu'on aurait pu attendre pour une pratique considérée comme un "mariage à l'essai ". Par la suite, des chercheurs ont constaté que la cohabitation a souvent très peu à voir avec le mariage et que ces deux formes d'union attirent des personnes dont les attitudes et les valeurs diffèrent au point de relever de cultures virtuellement distinctes.

Dans un article important où ils comparent les cohabitants aux célibataires et aux gens mariés, Rindfuss et Vanden Heuvel (1990) postulent que, eu égard au mariage, la "culture de la cohabitation " se caractérise par le refus de l'engagement et de

1 Ce phénomène n'est pas nouveau. Dans plus d'une société au cours de 1'histoire, les hommes pouvaient avoir une partenaire sexuelle régulière en plus de leur épouse légale: maitresse, concubine, etc. Le nombre d'unions de ce genre n'êtait pas forcèment négligeable, surtout dans les classes supérieures. Mais on imagine difficilement de nos jours une tentative pour en établir le compte à la faveur d'un recensement. De même, bien que, dans les pays industrialisés, de nombreux divorces soient de toute évidence hâtés par une " aventure " ou la rencontre d'un nouveau partenaire, ces événements figurent rarement dans les biographies recueillies par les enquêtes sur la famille. Cette discrétion, concevable dans le cas d'un organisme gouvernemental de collecte de données, est moins acceptable pour la démographie en tant que science des comportements. L'expérience du National Opinion Research Center avec l'enquête nationale américaine sur les comportements sexuels illustre le caractère embarrassant de la dépendance des sciences sociales à l'égard des gouvernements pour le financement de l'un de leurs principaux instruments de recherche. 
la permanence: "Ces dernières années, tout en continuant de s'investir dans des relations intimes, les gens se sont éloignés de l'engagement et de la permanence associés au mariage et à la famille " (p. 722). Mais si on ne le regarde pas par la lorgnette de la tradition, des lois et des idéaux, le mariage n'apparait en rien comme un état permanent, et moins que partout ailleurs aux États-Unis, où les taux de divorce par cohorte de mariage dépassent trente pour cent. Sans doute une proportion beaucoup plus forte d'unions hors mariage et de mariages précédés de cohabitation débouchent-ils sur une séparation, mais l'écart entre leurs taux de rupture et ceux des mariages actuels est probablement inférieur à celui qui sépare ces derniers des mariages conclus avant la hausse de la divortialité survenue au milieu du siècle.

Bracher, Santow, Morgan et Trussell (1993), commentant leur propre étude sur le divorce en Australie (menée à l'aide des données de la meilleure qualité et des méthodes d'analyse les plus avancées), font cette remarque qui décrit bien l'état de nos connaissances sur les formes actuelles d'union:

Si détaillés et exhaustifs que soient les facteurs " explicatifs " que nous avons eus à notre disposition, ils ne sont, somme toute, que de pâles reflets des facteurs probablement non mesurables qui cimentent les mariages ou les conduisent à la rupture; et il serait regrettable que la sophistication apparente des mesures le fasse oublier (p. 423; c'est nous qui soulignons).

Pour nous défaire de ce handicap, il importe de dépasser les approches démographiques classiques. Cela signifie :

- se mettre en marge des points de vue "bien-pensants", étudier les comportements tabous;

- s'intéresser davantage aux processus, et non pas seulement aux états;

- travailler sur des continuums multidimensionnels et non sur de simples catégorisations;

- accorder plus d'attention aux dimensions subjectives, aux significations;

- utiliser des outils quantitatifs "nouveaux": analyse factorielle et analyse de groupement; modélisation des systèmes; ensembles flous et modélisation floue; analyse séquentielle;

- recourir davantage aux méthodes qualitatives.

La transformation des formes d'union est si radicale qu'elle fait éclater les concepts et analyses du passé. La cohabitation 
est l'une des manifestations de la vaste révolution sexuelle qui a dénoué les liens entre sexualitê, mariage et reproduction, de concert avec le progrès technologique (notamment la contraception) et le relâchement des normes sociales. Les comportements sexuels et reproductifs et les modes de mise en union ressortissent maintenant du domaine privé autant que du domaine social, et leurs formes semblent se multiplier sans que les gouvernements, la religion, le milieu ou la famille aient le pouvoir d'endiguer le mouvement.

\section{UNIONS NON ÉPHÉMĖRES AVEC OU SANS PARTAGE DU DOMICILE : VUE D'ENSEMBLE}

La première difficulté que pose l'étude de la cohabitation a trait à la terminologie. À défaut d'élégance, le titre de cette section a l'avantage de couvrir le spectre des comportements qui nous intéressent, soit l'ensemble des relations à caractère sexuel un tant soit peu durables qui n'ont pas été sanctionnées par un mariage légal. Sont compris les couples homosexuels, les unions libres "ordinaires" et les cas où les partenaires n'habitent pas ensemble mais partagent régulièrement le même lit, à propos desquels certains recensements antillais utilisent l'expression "visiting relationships ${ }^{2}$.

Les termes adaptés à la multiplicité des formules ne sont pas faciles à trouver. En divers temps et lieux, on a parlé de companionate marriage (permettant à un couple sans enfant de divorcer par consentement mutuel, libre de toute obligation mutuelle), d'union de fait, de cohabitation, de couple non marié, d'union libre, de mariage coutumier ou d'union coutumière (common-law). Lors de ses premières tentatives pour cerner le phénomène, le U. S. Census Bureau a proposé le sigle POSSLQ (pour persons of the opposite sex sharing living quarters [personnes de sexe opposé partageant un logement]);

2 À propos des "unions sous deux toits ", voir Villeneuve-Gokalp, 1997. L'exclusion de ce type d'union sous prétexte qu'il n'y a pas partage d'un logement au sens du recensement témoigne d'un biais en faveur du mariage légal: les couples qui ne vivent pas dans le même ménage à cause de leur travail sont néanmoins considérés comme mariés, tout comme les couples qui font chambre à part et ceux dont le mari, étant chauffeur de poids lourds, passe le plus clair de son temps sur la route. Pourquoi faudrait-il que deux personnes non mariées ayant entretenu une relation prolongée et socialement reconnue ne soient pas considérées comme un couple pour la seule raison que de temps en temps chacune rentre chez elle? 
mais cette dernière catégorie est à la fois trop large, incluant certaines relations de cohabitation sans caractère sexuel, et trop étroite, puisque les relations entre personnes de même sexe sont exclues.

Nous avons, au Canada, une difficulté qui nous est propre. Le terme anglais utilisé dans les récentes enquêtes de Statistique Canada est "common-law union "; le terne français est "union libre". Comme nous l'avons signalé, l'un évoque un encadrement par la loi et l'État, l'autre la liberté. En outre, l'adjectif common comporte, comme d'ailleurs son correspondant français, des connotations péjoratives : " sans titre, statut ou rang particulier; sans distinction; moyen; de qualité médiocre ou inférieure, de deuxième ordre; doué de manières sans finesse ou grossières, vulgaire " (tiré de Nelson, Canadian Dictionary of the English Language, passim, traduction et ponctuation libres). Combien de Canadiens de langue anglaise savent que ce mot renvoie à la tradition anglaise du droit coutumier ou, en étant conscients, le perçoivent dans un sens positif ? Ne serait-ce qu'à cause des choix de vocabulaire nécessités par la traduction, on peut s'attendre à ce que les usagers des deux langues interprètent différemment le sens et la visée d'une même question.

Mais les problèmes de formulation peuvent aussi se poser à l'intérieur de la même langue. Dans l'Enquête sur la famille de 1984, Statistique Canada a interrogé les gens sur les

common-law partnerships, including those that led to marriage [les unions de droit coutumier, y compris celles qui ont conduit au mariage. Note : traduction quasi littérale, ici et dans les deux cas suivants. Le questionnaire français employait " union libre "].

Et on leur posait la question suivante :

Have you ever been a partner in a common-law relationship? By this we mean, partners live together as husband and wife without being legally married (Avez-vous déjà fait partie d'un couple de droit coutumier ? Cette dernière expression signifie que les partenaires vivent ensemble comme mari et femme sans être mariés légalement].

Or, la même année, l'Enquête sur la fécondité demandait :

Have you ever lived with a partner without being married? [Avezvous déjà vécu en couple sans être marié ?].

Pour les femmes en âge de procréer, les résultats comparables des deux enquêtes diffèrent de façon appréciable (Burch, 
1985). En ce qui concerne les unions en cours au moment des entrevues, les résultats sont semblables à tous les âges sauf les plus jeunes (18-19 ans): dans ce groupe, 5,7 pour cent des répondantes de l'Enquête sur la famille ont déclaré vivre "en union de droit coutumier ", " comme mari et femme ", etc., alors que 11,4 pour cent - le double - de celles de l'Enquête sur la fécondité ont dit "vivre en couple sans être mariées ". Pour une question sur les unions passées, les résultats des deux enquêtes présentent des écarts importants dans tous les groupes d'âge, étant en gros de dix points inférieurs dans l'Enquête sur la famille. Bien que les deux enquêtes aient essayé de cerner la même réalité, il apparaît qu'elles n'ont pas capté les mêmes types d'unions. La question de l'Enquête sur la famille semble connoter des unions plus "sérieuses " et plus traditionnelles. Les jeunes femmes sont-elles moins susceptibles de former de telles unions, ou moins portées à envisager leur couple sous cet angle? Les répondantes étaient-elles toutes moins susceptibles d'apparenter leurs unions précédentes au mariage ? On peut spéculer...

Si un vocabulaire diffërent peut servir à désigner la même réalité, ou des réalités différentes, il est également vrai que les mêmes mots - notamment cohabitation, union coutumière et union consensuelle - peuvent recouvrir des phénomènes distincts. Santow et Bracher (1998), dans un examen des tendances de la nuptialité préparé pour le congrès de 1'UIESP tenu à Pékin en 1997, citent un éventail de pourcentages de cohabitants allant de 44 pour cent des femmes de 20-24 ans en Suède en 1981 à 5 pour cent des Américaines du même âge. Or il est évident qu'un modèle de comportement adopté par près de la moitié des jeunes adultes d'un pays ne peut pas être considéré comme le même lorsqu'il est le fait d'une petite minorité. Il est "déviant " dans le second cas, en bonne voie de devenir "légitime ", acceptê comme norme voire recommandé dans le premier cas. À un moment donné, une différence quantitative de cet ordre devient une différence qualitative.

Plus loin, Santow et Bracher font ce commentaire : "Une raison importante de l'intérêt des démographes pour l'hétérogénéité est l'espoir de parvenir, en cernant les caractéristiques démographiques associées à la fréquence plus faible ou plus élevẻe d'un événement démographique particulier, à mieux connaitre les processus dont il est l'aboutissement " (p. 931). Mais lorsqu'un taux de prévalence est neuf à dix fois supérieur à un autre, peut-on encore dire que les deux mesurent un "événe- 
ment démographique particulier " au sens où on l'entend pour une naissance ou un décès ? Certes non ${ }^{3}$.

Ce point faible est aussi celui des enquêtes biographiques et des méthodes d'analyse multi-états qui servent à en exploiter les données. Ces démarches, qui rallient actuellement beaucoup de chercheurs, ont rendu un fier service à la démographie en stimulant l'intérêt pour l'analyse dynamique des microphénomènes et pour la notion de processus, mettant notamment en évidence la diversité des itinéraires susceptibles d'aboutir au même point. Mais elles risquent de rater la cible dans la mesure où elles laissent de côté des états importants mais difficiles à conceptualiser ou à mesurer, ou encore attribuent un même état à des individus qui, après un examen plus approfondi ou plus minutieux, seraient affectés à des états différents. Tout en reconnaissant que l'analyse multi-états nous fait faire un grand pas en avant, il nous faut admettre que les modèles qu'elle engendre peuvent produire, non pas seulement un "pâle reflet " de ce qu'ils sont censés représenter (pour en revenir à Bracher, Santow, Morgan et Trussell [1993]), mais encore une image distordue.

Quels comportements, outre la cohabitation elle-même, devons-nous inclure dans notre éventail pour embrasser tout le phénomène? Roussel (1984), l'un des premiers chercheurs à avoir tenté de dégager des critères, utilise ceux clu statut légal et de la présence d'un enfant, et distingue entre l'union libre (couple non marié ayant un enfant) et la cohabitation (homme d'au moins 25 ans et femme célibataires vivant sous le même toit). La frontière est tracée entre les unions d'une certaine solidité et les unions plus éphémères.

Villeneuve-Gokalp (1990) distingue pour sa part cinq profils types de cohabitants, basés sur "le premier événement qui suit la mise en couple (mariage, naissance ou séparation) " et sur des critères de durée. La cohabitation peut être :

3 Santow et Bracher, conscients de la difficulté, se tournent bien vite vers " d'autres aspects de la comparabilité et de l'interprétation des résultats d'enquête " (p. 931). Ces problèmes de concepts et de définitions ne sont pas nouveaux en démographie. Nous avons systêmatiquement échoué à conceptualiser et à mesurer adéquatement l'événement démographique migration, comblant les failles de nos méthodes par des conventions largement arbitraires avec lesquelles nous avons fini par nous sentir à l'aise. Subsiste donc une immense faiblesse à ce chapitre en démographie comparative puisque, à défaut de mesure disponible, il nous faut emprunter toutes sortes de détours pour classer les populations au point de vue de l'incidence de la migration ou du degré de mobilité. 
1) un prélude au mariage, débouchant logiquement sur ce dernier;

2) un test avant le mariage (étendu sur plus d'un an);

3) une union éphémère (de durée inférieure à un an);

4) une union stable sans engagement (n'impliquant ni mariage ni enfant, mais prolongée);

5) une union libre (le couple a un ou des enfants, mais ne se marie pas).

C'est là une typologie intelligente et nuancée, sans doute adaptée à la situation française de l'époque, et utile au sens où l'entend Roussel (1984: 151) :

Une typologie en effet est utile dans la mesure où elle n'est pas un simple artifice de classement et où elle permet de comprendre l'unité logique de comportements dont on avait d'abord seulement constaté qu'ils allaient ensemble. Dans ce sens, le modèle transforme le constat en discours intelligible.

Mais jusqu'où aller ? S'il existe cinq types de cohabitation, on pourrait sûrement trouver, pour revenir à la typologie classique des états matrimoniaux, divers types de célibat, de mariage, de séparation, de veuvage et de divorce. Certaines personnes sont célibataires pour de bon; d'autres espèrent ne pas le rester. Certains mariages sont solides; d'autres tiennent bon tant que l'un des conjoints n'a pas noué d'autre relation amoureuse. En Italie, des chercheurs ont défini une catégorie "séparation sous le même toit " pour les cas où le mariage et le partage du logement subsistent pour des raisons d'affaires ou des motifs familiaux, tandis que les conjoints recherchent l'intimité au dehors (De Rose, 1992). Voilà que notre typologie de quatre ou cinq catégories s'est élargie à une vingtaine, posées a priori, mais défendables ${ }^{4}$. Nous pourrions écarter les

L'utilité de typologies contenant un aussi grand nombre de catégories est un intéressant problème de méthodologie. A première vue, la multiplication des catégories semble relever d'une tendance à particulariser et à créer des formes logiques à la chaîne, voire à établir des distinctions pour le plaisir de la chose. Un bulletin de nouvelles récent nous apprenait qu'un chercheur canadien (en sciences sociales) avait découvert (?) vingtcinq types de mères et neuf types de pères. Mais cette activité scientifique, comme beaucoup d'autres, est marquée par la tension permanente entre le souci du détail et l'analyse d'une part et la généralisation et la synthèse d'autre part. Le problème est posé de manière particulièrement aiguë par le nouveau travail de description des événements jalonnant les parcours biographiques, où des catégories pourraient résulter de la permutation de tous les états possibles. 
quelques-unes qui regrouperaient peu de cas, mais ce serait une décision arbitraire, et malheureuse si certaines devaient signaler le début de nouvelles tendances : vers 1970 , le nombre de cohabitants était faible dans des pays comme le Canada, car le phénomène de l'union libre ne faisait que s'amorcer.

Certaines recherches empiriques ont commencé à éclairer la complexité des comportements actuels de mise en couple.

Kravdal (1997), à l'aide des données d'une enquête menée en 1988 auprès de 4019 Norvégiennes, constate une forte hétérogénéité parmi les couples cohabitants, même lorsqu'ils ont eu un enfant : " il y a trois catégories équivalentes de naissances chez les couples cohabitants: les naissances mal planifiées (c'est-à-dire désirées, mais survenues trop tôt, auxquelles s'ajoute un petit nombre de naissances non désirées); les naissances planifiées par des couples qui projettent de se marier; et les naissances planifiées par des couples qui n'entendent aucunement se marier " (p. 269). Parmi les couples de la dernière catégorie, certains voyaient le mariage lui-même comme un obstacle, en raison surtout des dépenses qu'il entraîne: la cohabitation est "pour l'homme "pauvre" ou trop "occupé" le substitut d'un mariage dont la précarité n'aurait pas été moindre" (p. 282) ${ }^{5}$. D'autres craignaient que la formalisation de leur relation en diminue la valeur humaine. Ils étaient nombreux aussi à apprécier la possibilité de rompre une union consensuelle assez facilement par comparaison avec un mariage légal si des difficultés ou l'apparition d'un autre partenaire devaient les mener à une séparation. Les couples présentés par Kravdal sont sérieux, prennent un certain type d'engagement (sans promettre de rester ensemble "jusqu'à leur mort"), ont des enfants : seules manquent les formalitês légales.

L'étude de Lillard, Brien et Waite (1995) confirme catégoriquement l'opinion selon laquelle les taux de divorce supérieurs des mariages précédés de cohabitation sont en grande partie un effet de sélection. Mais si l'on élimine cet effet, le " test " de la cohabitation ne semble pas abaisser les risques de divorce. Les auteurs ont leur explication :

5 Cette remarque intéressante atteste la permanence d'un modèle qui a existé par le passé en Amérique latine, mais aussi en Europe, à savoir la cohabitation en lieu de mariage chez les pauvres et les marginaux. Il y a toujours des mal nantis parmi nous, en particulier chez les jeunes. Sans l'aide de leur famille, ces derniers n'ont pas toujours les moyens de s'off:-ir un mariage "convenable " et certains ne voient pas l'utilité d'une cérémonie civile sans éclat à seule fin de légaliser leur union. 
l'expérience de la cohabitation modifie l'opinion des couples sur le mariage, leur fait prendre leurs distances par rapport à l'institution et les rend plus enclins à divorcer. La stabilité du mariage peut être diminuée par ce changement d'attitude, même si elle est accrue par la conscience plus nette que l'on est bien assorti" (p. 455; souligné par les auteurs).

Et ils complètent cette hypothèse par une autre : " la cohabitation peut différer du mariage dans sa structure et ses attentes [...] au point que linformation acquise par les conjoints durant la cohabitation ne soit pas particulièrement utile pour prédire ce que sera leur mariage " (p. 455).

Finalement, les chercheurs soulignent avec insistance que chaque union s'inscrit dans un processus et qu'il importe de dépasser l'examen détaillé de décisions ou événements isolés : " il faut accorder beaucoup d'attention aux liens entre les décisions, en l'occurrence celle de cohabiter avant le mariage et celle de rompre celui-ci (p. 455; souligné par les auteurs).

Dans un contexte culturel très différent, Castro Martin (1997) constate qu'en Amérique latine les "mariages sans papiers " résultent d'un mélange complexe de traditions et d'adaptation aux circonstances. Son commentaire est clair :

Malgré leur plus grande instabilité, beaucoup d'unions non légalisées durent longtemps et ne peuvent donc pas être qualifiées avec justesse d'étape de transition dans le parcours matrimonial. Fait plus important, les unions consensuelles sont le cadre dans lequel sont habituellement conçus et êlevés les enfants, ce qui brouille la distinction entre mariage de droit et mariage de fait pour ce qui est de la reproduction. Il s'ensuit que l'attention traditionnellement accordée au mariage légal en démographie n'est de toute évidence pas adaptée à la description du processus de formation des familles et de reproduction tel qu'il existe dans cette région (p. 956).

\section{DIMENSIONS MULTIPLES ET ENSEMBLES FLOUS : LES APPROCHES QUANTITATIVES}

Les typologies reposent sur des dimensions nombreuses, explicites ou non, mais procèdent souvent de postulats arbitraires, stéréotypés sur la distribution de ces dimensions, le plus habituel, à la fois puissant et implicite, étant qu'elles varient simultanément de façon marquée. Il en résulte des types discrets : gemeinschaft-gesellschaft; rural-urbain; marié- 
cohabitant ${ }^{6}$. La compréhension des formes d'union actuelles nécessiterait la réalisation d'un programme de recherche empirique qui a) reconnaîtrait dans toute son étendue la multidimensionnalité des phénomènes à saisir et b) explorerait les méthodes empiriques permettant de ramener cette multidimensionnalité à des niveaux opératoires ?

Une liste définitive des dimensions qui concernent les unions nécessiterait un inventaire systématique. Les éléments suivants esquissent quelques contours :

- la composition selon le sexe : mixte; deux femmes; deux hommes;

- l'exclusivité (forme de base) : monogamie; polygynie; polygamie;

- la fidélité : forme de base adoptée de façon stricte ou tolérance à l'égard des " aventures";

- le désir d'avoir et d'élever des enfants;

- le désir de permanence, la permanence effective (la durée, au sens de la démographie);

- l'engagement des partenaires l'un envers l'autre et envers la relation;

- le choix du partenaire : libre, ou union arrangée ou forcée;

- en cas de libre choix du partenaire, les motifs : amour ou convenance (recherche d'un partenaire sexuel qui soit aussi un compagnon pendant l'année scolaire; désir de plaire aux parents; d'avoir droit à des prêts étudiants plus élevés ou à quelque autre paiement de transfert; etc. Il existe des mariages mais aussi d'autres unions de convenance);

- le caractère public ou secret (clandestin) de la relation (s'il s'agit d'une maîtresse, d'une concubine, d'un petit ami, etc., compte tenu des degrés de clandestinité);

6 Mème lorsqu'on fonde des typologies sur des continuums bipolaires ou sur des idéaux-types, on a tendance à négliger les cas qui se situent entre les extrêmes pour au moins une dimension pertinente.

7 Le caractère opérationnel ou adéquat d'une typologie dépend de l'objectif poursuivi. Les scientifiques cherchent à distinguer les cas le plus nettement possible du point de vue du pourquoi et du comment. Les gouvernements et les administrations ont parfois d'autres impératifs; ainsi, une typologie des "groupes sociaux " utilisée lors du recensement canadien de 1996 pour fournir au gouvernement les données nécessaires à la préparation d'une loi sur l'équité en emploi n'en était pas moins scientifiquement contestable. 
- l'existence et la nature d'un rite inaugural: cérémonie purement privée ou réunissant amis, famille, communauté, dignitaires, magistrats, ministres du culte, etc.;

- le statut légal : union légale au sens strict; union reconnue par la loi (telles les unions coutumières au Canada); union illégale;

- le mode de dissolution de l'union et la plus ou moins grande facilité de la rupture : décision individuelle ou de couple; autorisations à obtenir (de l'Église, d'un juge, etc.), procédures à engager;

- les droits ou privilèges subséquents à la dissolution de l'union: partage des biens, garde des enfants et droits de visite; pensions alimentaires; égalité devant la loi (en Ontario, un cohabitant n'hérite pas de son conjoint mort intestat).

La liste est longue, sinon infinie. Comment en tirer parti ?

D'abord, il faut recueillir des données sur chacune des dimensions. Étant donné les pratiques actuelles, la chose est facile pour certaines, mais non pour d'autres. Statistique Canada n'a pas réussi, par exemple, à dénombrer les couples de même sexe au recensement de 1996, parce que des essais infructueux, s'ajoutant aux réactions contradictoires des homosexuels eux-mêmes, ont mené à la décision de ne pas poser de question en ce sens. L'information sur certaines dimensions est inséparable du statut légal, qui peut impliquer la nécessité de divorcer, un droit de regard de la justice sur les biens et les enfants. La plus grande difficulté a trait au domaine des intentions et des significations. Les enquêtes démographiques à grande échelle ne sont pas allées très loin à cet égard. Elles ne sont d'ailleurs pas particulièrement bien adaptées à la tâche comparativement aux approches plus intensives de cueillette de données. Il faut combiner les deux démarches : utiliser entrevues intensives, entretiens en profondeur avec groupes cibles et autres méthodes permettant des investigations fouillées (McNicoll [1992] parle à ce propos des "profondeurs quasi illimitées de l'analyse et de l'explication des comportements"), puis se servir des résultats pour mieux formuler les questions simples et directes destinées aux recensements et aux grandes enquêtes.

Il s'agit maintenant, bien sûr, de réduire les données rassemblées sur l'ensemble des dimensions énumẻrées (et d'autres encore...). Il serait possible à ce stade de mesurer les 
variables en procédant à une analyse factorielle afin de voir si la variation des caractéristiques des unions peut être captée par un nombre réduit de dimensions plus générales. Pour des raisons qui demeurent obscures, la démographie a tendu à se détourner de cette méthode ou des autres méthodes éprouvées de mesure et de classification, leur préférant des indicateurs relativement concrets. Mais il existe des exceptions. En demographie de la famille, Benoît, Lévi et Vimard (1993) ont montré qu'il est possible de réduire un grand ensemble de caractères des ménages à quelques dimensions liées au nombre d'enfants, au nombre d'adultes et au nombre de couples mariés ${ }^{8}$. De même, Hall (1993) a recouru aux procédures de classification de l'analyse factorielle pour mesurer ce qu'il appelle "l'individualisme reproductif "et son impact sur la dissolution des mariages. Mais il a été contraint de conserver le couple mariage-union libre de Statistique Canada pour distinguer les types d'unions.

$\mathrm{Si}$ nous disposions d'une abondance de données sur les caractéristiques des unions, il serait sans doute possible d'en dégager, au moyen de procédures de classification adéquates, des dimensions explicatives moins nombreuses dont les scores seraient ensuite soumis à des analyses multivariées, ce qui permettrait par exemple d'étudier l'effet d'une variable comme l'engagement sur la durée des unions. C'est pratique courante - et fructueuse - en psychologie, mais non en démographie, où l'élaboration des concepts et des mesures tend à prendre une forme plus discursive et parfois improvisée.

Pour créer les typologies dont nous estimons avoir besoin, il serait souhaitable d'appliquer l'analyse de groupement à de grands ensembles d'unions très diversifiées afin de disposer de catégories qui soient calculées empiriquement au lieu de reposer sur des considérations purement légales ou des conventions. Ainsi, il se peut fort bien qu'empiriquement certaines unions libres soient, légalité mise à part, plus proches du mariage que d'autres. Les démarches de classification actuelles ne font pas ressortir ce fait important. Certes, la taxinomie vise avant tout à créer des catégories assez homogènes. Mais les classifications basées sur un ou deux critères, tel le statut

8 Burch et Madan ont obtenu des résultats similaires avec des données sur les ménages canadiens (travaux inédits). Voir aussi Burch (1991), qui utilise l'analyse factorielle pour dégager les dimensions où s'exerce la subjectivité : normes perçues, valeurs, préférences personnelles, etc. 
d'une union devant la loi, peuvent précisément donner le résultat contraire.

Le désintérêt relatif de la démographie pour les procédures automatiques de mesure et de classification ou de groupement pose une intéressante question à la sociologie des sciences ${ }^{9}$. Les logiciels qui les rendent accessibles sont pourtant à portée de main. Le progiciel SPSS, par exemple, contient des procédures comme Alscal, Cluster, Factor, Proximities et Quick Cluster, qui toutes peuvent se charger de réduire les dimensions à des ensembles moins grands ou d'affecter les cas à un nombre limité de catégories calculées empiriquement. Abbott (1988), imputant le succès de certaines procédures statistiques à leur inclusion dans des progiciels très répandus comme SPSS ou SAS, explique par les aléas de la "commercialisation " l'abandon relatif où sont laissées les méthodes d'équations différentielles proposées par James Coleman dans son Introduction to Mathematical Sociology (1964), eu égard à la popularité de la régression multiple prônée par Blalock (1960). Cette explication ne vaut certes pas dans le cas qui nous occupe, car les méthodes mentionnées ci-dessus sont "disponibles dans le commerce " depuis plus de vingt-cinq ans ${ }^{10}$.

Convient-il de mettre les cohabitants dans la case célibataire ou dans la case marié? Il faut des démarches empiriques novatrices pour dénouer cette question, qui ne se tranche pas comme un nœud gordien.

$\mathrm{Ne}$ faut-il pas en effet s'incliner devant le fait que les unions, comme les gens, ne se rangent pas forcément sans ambiguïté dans une seule case bien nette ? Peut-être appartiennent-elles à des ensembles flous. Comme d'autres découvertes, la logique floue et ses applications en modélisation sont

9 Pour une application récente de la classification hiérarchique automatique à la comparaison de la mortalitê dans divers pays, voir Bourbeau et Courville, 1997.

10 Abbott ne dépasse pas 1985 dans son examen de la popularité respective de la régression multiple et des équations différentielles en sociologie. Plus récemment, les secondes sont arrivées " sur le marché ", incorporées à des progiciels comme Mathematica, Mathcad, Derive, Scientist, Macsyma, etc., et à des logiciels de modélisation des systèmes tels que Dynamo, Stella II, Modelmaker, Vensim, Matlab, etc. Encore là, ces outils ne sont guère utilisés par les démographes. Les économistes ont d'autres instruments de " modêlisation dynamique " et cultivent habituellement un plus grand nombre dhabiletés techniques diverses. Mais la démographie sociale demeure tournée vers des méthodes qui descendent en droite ligne de la régression multiple, tels les modèle à risques proportionnels et la régression logistique. 
largement méconnues par le courant dominant de la démographie, même si certains appels intuitifs sans équivoque se font entendre en leur faveur et malgré la "commercialisation " de certaines ressources ${ }^{11}$. Les enfants confiés à la garde conjointe de leurs parents séparés appartiennent à deux ménages, fût-ce à des degrés différents; mais dans les recensements et les enquêtes on doit habituellement les attribuer à l'un ou à l'autre (il est vrai que Statistique Canada a entrepris de faire face à ce problème dans l'Enquête sociale générale de 1995). De même, certaines personnes mariées entretiennent une relation de couple avec un second partenaire, si bien qu'elles sont à la fois mariées et cohabitantes. Mais ce peut être à des degrés différents. L'une peut avoir emménagé avec le second partenaire sans attendre le divorce, êtant alors plus cohabitante que mariée; une autre peut vivre la plupart du temps dans la résidence familiale tandis que se poursuit sa relation avec le second partenaire, lequel n'appartient pas au même ménage qu'elle : elle est plus mariée que cohabitante. Compte tenu de certains aspects pertinents, on fausse la réalité en voulant à tout prix classer un cas dans une seule catégorie.

L'analyse séquentielle est une autre approche quantitative prometteuse qui pourrait nous aider à mieux comprendre la nature et la dynamique des unions, même à partir des catégories actuelles de recensement et d'enquête (Abbott, 1983, 1995). La signification du mariage ou de la cohabitation peut être éclairée par les événements qui les précèdent ou les suivent. Des analyses empiriques récentes révèlent l'hétérogénéité croissante des itinéraires individuels. Ravanera, Rajulton et Burch (1998), par exemple, nous apprennent que, dans les cohortes récentes, seulement une faible fraction des répondants (environ un tiers) peut être répartie dans six parcours plus ou moins traditionnels; les deux tiers ont emprunté une trajectoire différente, plus complexe. Ce constat met en lumière la nécessité des méthodes de réduction des données dont nous avons parlé. Pour ce qui est de la comparaison et de la réduction des parcours ou séquences, des articles récents d'Abbott (1995) et de Billari et Piccarreta (1999) présentent les méthodes les plus intéressantes.

11 Le populaire progiciel Mathematica peut s'accompagner d'un logiciel appelé Fuzzy Logic, qui permet la modélisation en logique floue. Liao (1989) a publié l'un des quelques articles qui explorent cette avenue. Voir aussi Cerbara, 1997. 
La démographie a trop tardé à adopter de nouvelles méthodes quantitatives mieux adaptées à la multidimensionnalité et à la dynamique complexe des unions, s'en tenant à des procédures que Hakkert (1992), à cause de leur simplicité et de leur caractère rétrograde, qualifie de "paper and pencil algorithms ". Mais il faut pousser plus loin, et en arriver à une démographie des comportements qui marie le qualitatif au quantitatif.

\section{PROCESSUS ET SIGNIFICATIONS : LES APPROCHES QUALITATIVES}

Selon Louis Henry, "le point de vue quantitatif est absolument prépondérant en démographie, au point qu'il n'y a pas de démographie sans chiffres ni statistiques" (Henry, 1972: 15). S'il est vrai qu'en démographie aucune recherche ne peut, par définition, évacuer le point de vue quantitatif qui est au cœur de la méthode, nous ferons valoir ici l'utilité des démarches qualitatives pour éclairer la cohabitation et les autres formes d'union. Ces méthodes semblent particulièrement adaptées à l'étude des unions en tant que processus, dont nous avons souligné la nécessité. La recherche qualitative met à profit un éventail étendu d'approches théoriques et d'instruments destinés à la cueillette, à l'analyse et à l'interprétation des données. Mais malgré leur diversité, la plupart des recherches qualitatives présentent un certain nombre de points communs :

- les processus et les significations sont au cœur de la démarche;

- la nature et la diversité de ces processus et significations sont pleinement reconnues;

- les observations et les analyses produites sont soigneusement contextualisées dans le temps et dans l'espace (Sabourin, 1993);

- le caractère socialement construit de la réalité, la relation intime entre le chercheur et son objet d'étude et les contraintes de situation qui donnent forme à la recherche ne sont jamais perdus de vue (Denzin et Lincoln, $1998: 8$ );

- le processus de recherche est circulaire : la cueillette des données, l'analyse et l'interprétation sont concomitantes et interreliées, au lieu de se succéder de manière linéaire; l'analyse des données et la construction de la théorie s'ali- 
mentent mutuellement, comme le suppose le concept de "théorie ancrée " (grounded theory : voir Strauss et Corbin, 1998);

- la poursuite des travaux est sous-tendue par une réflexion épistémologique sur la nature de la recherche scientifique en sciences sociales.

La liste pourrait s'allonger, mais elle suffit à montrer que la démarche qualitative est de nature à favoriser l'étude du mariage et de la cohabitation dans l'optique que nous avons proposée, c'est-à-dire en tant que continuums multidimensionnels déployés dans le temps.

Les recherches menées en démographie à l'aide de l'approche qualitative ont exploité une combinaison de méthodes qualitatives et quantitatives, comme lillustrent certaines de leurs caractéristiques :

- utilisation d'un plan quasi anthropologique pour étudier les comportements et construire le cadre théorique (Caldwell, 1982; Caldwell, Reddy et Caldwell, 1988);

- entrevues en profondeur avec de petits groupes cibles pour établir les catégories destinées à un questionnaire d'enquête ou pour trouver ou préciser l'interprétation d'un résultat issu d'une analyse quantitative (sens d'une relation, signification sociale douteuse d'un résultat statistiquement significatif) (Kertzer et Fricke, 1997; Podhista et al., 1990);

- étude des milieux où apparaissent les comportements démographiques, afin d'explorer les processus qui y conduisent et le sens donné à ces derniers; la culture est au cœur de ces études (Fricke, 1997);

- utilisation de méthodes historiques à caractère qualitatif (recherche d'archives) pour comprendre les comportements démographiques cernés par la démographie historique (Kertzer, 1993);

- recours à des théories se rapportant à l'approche qualitative pour dresser une critique de la démographie comme science (Greenhalgh, 1996, 1997).

On le voit, les chercheurs ne s'en sont pas tenus à une démarche purement qualitative. Comme Knodel (1997), nous croyons d'ailleurs que les démographes qui s'intéressent à certains outils qualitatifs n'ont pas à aller sur le terrain à la manière des anthropologues. S'il est vrai que ces derniers peuvent leur fournir des résultats utiles pour comprendre les 
comportements démographiques, les démographes vont quand même continuer à travailler sur des grands ensembles de données agrégées recueillies par d'autres personnes. En ce qui concerne l'étude de l'union libre, l'approche qualitative peut être mise à profit à divers moments du processus de recherche, pour diverses explorations :

- recherche axée sur des aspects cognitifs : utilisation du langage; signification et interprétation des mots, évolution de leurs connotations, sens qui leur sont rattachés par les diverses cohortes, par les deux sexes, par les groupes socioéconomiques et ethniques. Cette approche peut être d'un grand secours pour formuler ou traduire des questions d'enquête ${ }^{12}$.

- recherche exploratoire sur les comportements, considérés comme des processus : détermination des parcours individuels et des événements liés à certains comportements, telle la cohabitation;

- recherche contextualisée : étude du comportement individuel dans le contexte de la famille ou du milieu; par exemple, rôle des relations de parenté et des autres types de relations dans la formation et la dissolution des unions (interactions entre biographies);

- examen des interprétations des gens à l'égard de leurs propres comportements: la signification accordée à diverses formes d'union pourrait conduire à des typologies comprenant plusieurs classes de mariage et de cohabitation basées sur la nature des relations, l'engagement, la présence ou l'absence d'enfants, la mise en commun ou la séparation des ressources, les stratégies d'adaptation au marché du travail, etc.;

- recherches plus "pointues" (sur de petites sous-populations, par exemple couples cohabitants formés de deux étudiants éloignés du foyer parental pour la première fois, ou de deux divorcés avec enfants; etc.);

- investigations sur des sujets tabous difficiles à cerner en enquête : comportements sexuels et réseaux sexuels; relations extraconjugales, etc.

12 Statistique Canada s'est servie d'entrevues en profondeur avec des groupes cibles pour préparer les questions destinées aux recensements et à ses grandes enquêtes sur échantillon. Mais, sauf erreur, les résultats de ces séances n'ont pas été publiés sous forme de "données". 
La collecte de données textuelles (qualitatives) à l'occasion d'entrevues individuelles ou de groupe devient ainsi un moyen d'ajouter une composante qualitative à une recherche démographique, avant, pendant ou après la cueillette ou l'analyse des données quantitatives. Cette composante peut servir à générer des hypothèses et mener à la conception d'une proposition de recherche et d'un questionnaire; elle peut également faire suite à une analyse quantitative, pour en clarifier ou en nuancer les résultats.

Jusqu'ici, en démographie, la plupart des chercheurs qui ont utilisé des méthodes qualitatives se sont intéressés à des cultures étrangères plutôt qu'à la leur: Fricke a étudié le Népal, Caldwell le Nigeria, Knodel la Thailande, Greenhalgh la Chine et Kertzer l'Italie. Il semble que nous n'ayons pas perçu l'utilité de l'approche qualitative pour l'étude des significations et des processus propres à nos sociétés. Peut-être la difficulté que nous posent les nouvelles formes de conjugalité offre-t-elle à la démographie l'occasion de passer, comme l'anthropologie avant elle, de l'étude de l'autre à l'étude de soi.

Finalement, on ne saurait passer sous silence l'avènement des logiciels de gestion des donneees qualitatives, qui accroissent nos possibilités d'analyse et débrouillent l'écheveau que représente encore le traitement des données textuelles (Tesh, 1992; Richards et Richards, 1998). Ils permettent en outre de mettre en relation les caractéristiques individuelles stockées dans une banque de données quantitatives et le contenu d'une banque de données textuelles. Les chercheurs désireux de marier qualitatif et quantitatif ont ainsi une corde de plus à leur arc ${ }^{13}$. Les logiciels permettent de revenir sur toutes les étapes du processus et de sauvegarder celles-ci, de sorte que les analyses sont disponibles pour la communauté des chercheurs. Tout en protégeant la vie privée de leurs informateurs, ceux-ci ont, via Internet, la possibilité de partager les bases de données textuelles constituées de réponses à des questions ouvertes, comme on le fait pour les données d'enquête.

"Il est de plus en plus admis, écrivait Preston il y a cinq ans, que des recherches mieux conçues, associant données quantitatives et données qualitatives, sont indispensables à la réalisation des objectifs de presque toute recherche sociale, même en démographie" (Preston, 1993 : 598; c'est nous qui

13 La version 4.0 de Qsr Nud*ist offre cette possibilité et peut donc lire les données quantitatives provenant de progiciels comme SPSS. 
soulignons). Faisant référence aux travaux de Caldwell, Knodel et Massey, il ajoute : "il me semble que cette intégration des pratiques a progressé davantage en démographie que dans tous les autres champs des sciences sociales" (ibid.). À la même époque, les principales universités américaines qui offrent des programmes de Ph.D. en démographie ont institué, en collaboration avec la Fondation Mellon, un programme conjoint de démographie et anthropologie, dans le but d'assurer le développement des théories et méthodes des deux disciplines. Brown University, l'un des leaders de ce mouvement, a organisé en 1993 un congrès de démographie anthropologique ${ }^{14}$ et, plus récemment (en novembre 1997), un séminaire sur la conception de programmes de formation à la recherche en démoanthropologie. Lors de ces rencontres, des nouveaux diplomés et des étudiants ayant reçu leur formation dans le cadre des programmes conjoints ont souligné les réticences des revues de démographie à publier leurs travaux qualitatifs. La place extrêmement modeste des êtudes à dimension qualitative dans les grandes revues spécialisées donne à penser que, malgré l'optimisme de Preston, l'approche qualitative des comportements démographiques fait son chemin péniblement. L'étude de la cohabitation nous donne l'occasion de vérifier de plus près l'utilité de cette approche pour l'analyse démographique classique et d'éclairer davantage les transformations rapides auxquelles sont soumis les rapports de couple, ici et ailleurs.

\section{CONCLUSION}

La démographie a atteint la maturité au XXe siècle, en symbiose avec l'État nation moderne et la statistique officielle, puis avec les organisations internationales comme les Nations Unies. Durant cette période, la mesure des grandes variables démographiques (taille des populations, croissance, structure par âge, fécondité, mortalité, migration), l'analyse de leurs interrelations, la modélisation, la prévision et même l'explication des comportements ont progressé à vive allure.

D'autres comportements et variables qui intéressent la démographie ont résisté à ses analyses classiques, en particulier dans le champ ouvert par l'étude du mariage, de la famille et des ménages. Aucun domaine ne jette sur ces difficultés une clarté plus crue que celui des nouvelles formes d'union. La

14 On trouvera les communications dans Fricke et Kertzer (1997). 
révolution des comportements sexuels et reproductifs qui s'est amorcée au cours des années 1960 dans les pays développés, et se répand maintenant par têtes de pont dans le monde, met les démographes au défi d'élargir leurs horizons et d'enrichir leur panoplie de théories et de méthodes.

La réalisation de cette commande nécessitera l'utilisation des nouveaux modes d'analyse rendus possibles par l'ordinateur, qu'il ne faudra plus réserver à l'exécution des anciens algorithmes. Elle exigera aussi l'exploitation des approches connues sous le nom de "méthodes qualitatives ", familières aux. historiens, sociologues et anthropologues, mais méconnues des démographes du courant dominant. Unies étroitement, plutôt que substituées, aux démarches quantitatives propres à la démographie, elles seront à l'origine des découvertes les plus marquantes.

Il ne s'agit pas de tourner le dos à un bilan plus que respectable, mais de l'utiliser comme tremplin et de l'accroitre. Nous n'avons aucunement voulu sous-estimer ici le progrès somme toute rapide des connaissances sur la cohabitation, le mariage et le divorce depuis le début des changements révolutionnaires des années 1960 et 1970, au Canada et dans beaucoup d'autres pays industrialisés. À peine quinze ans - une demigénération peut-être - se sont écoulés depuis que l'Enquête sur la famille et l'Enquête sur la fécondité ont fourni aux chercheurs les premières données détaillées sur la cohabitation, sous forme d'information biographique. Cette information a été mise à jour régulièrement par l'Enquête sociale générale et peut être complétée, à certaines fins, par les données sur l'union libre fournies par le recensement canadien depuis 1986. Statistique Canada a su trouver, avec une rapidité remarquable, des moyens pour observer l'apparition des nouveaux modèles de comportement en matière de formation et de dissolution des unions, tout en respectant les lois, la sensibilité de l'opinion publique et les exigences statistiques de continuité dans les séries de données.

Durant la même période, de nouvelles méthodes se sont répandues, notamment celles qui permettent l'analyse des données issues des récits biographiques. L'application des tables d'extinction à ces données est désormais pratique courante et lève l'hypothèque due aux sorties d'observation. L'extension des modèles à risques proportionnels a permis d'exploiter cet avantage dans l'analyse multivariée et d'étudier les facteurs (les variables stochastiques) associés à l'existence ou à. l'inexistence 
et au calendrier des événements (âge ou durée du mariage ou de la cohabitation). L'analyse multi-états permet de décrire les états et itinéraires individuels ou collectifs qui se succèdent au long des étapes du cycle de vie, notamment ceux qui correspondent à la formation et à la dissolution des unions et des familles.

Nous connaissons donc assez bien le nombre de Canadiens qui cohabitent à divers moment de leur vie et l'issue de ces unions : maintien, mariage, rupture. Nous savons que la plupart des unions libres prennent fin assez tôt (en moins de dix ans), par un mariage ou une séparation. Nous savons que, dans l'ensemble, nombreux sont les Canadiens à opter pour la cohabitation à un moment ou l'autre de leur vie, surtout par rapport aux époques qui nous précèdent, même si la cohabitation est loin d'être universelle et ne constitue même pas un modèle majoritaire. Nous savons qu'elle est plus répandue dans une région (le Québec) que dans les autres, ainsi que dans des sous-groupes particuliers : elle est très fréquente chez les gens qui ne pratiquent aucune religion, rare dans certaines communautés ethniques (d'origine italienne ou chinoise notamment).

Nous savons que les mariages précédés de cohabitation risquent davantage de se terminer par le divorce, et que cette probabilité plus élevée est surtout attribuable, non à une relation de cause à effet, mais à un effet de sélection : les personnes enclines à cohabiter sont également celles qui sont susceptibles de divorcer. Nous savons que la reproduction reste associée au mariage, même si de plus en plus de femmes en union libre ou sans partenaire régulier ont un enfant.

Ce bagage de connaissances est appelé à augmenter, car les données déjà disponibles n'ont pas encore été intégralement analysées, et d'autres matériaux viennent s'y ajouter, grâce notamment aux diverses enquêtes longitudinales de Statistique Canada.

Mais il doit être perfectionné et approfondi par les moyens dejjà suggérés. Une fois que nous avons classé les répondants des recensements et enquêtes comme cohabitants ou vivant en union libre ou mariés, que pouvons-nous dire de plus : que savons-nous du type de personnes dont il s'agit, ou de leurs attitudes et de leurs intentions à l'égard de leur union? "Marié " rime avec mariage légal, mais tous les mariages ne sont pas similaires pour ce qui est des dimensions non légales, et nous n'avons pas beaucoup de données sur ces dernières. Un mariage traditionnel avec cérémonie religieuse et invités des 
deux familles se compare-t-il avec un mariage dont les conjoints ont réglé eux-mêmes le scénario (échange des anneaux au salon ou sur la plage, un prêtre, ministre ou magistrat, quelques intimes) ? Que dire d'une union libre dans laquelle les conjoints s'engagent avec la ferme intention de toujours rester ensemble et d'avoir des enfants, parce qu'ils contestent par principe l'autorité de l'Église ou de la loi sur le mariage légal, ou d'une union de convenance par laquelle des étudiants organisent leur vie sexuelle et s'assurent une présence amie durant leurs années d'université ? Si les deux peuvent être semblables au regard de la loi et entrer dans la même catégorie d'enquête, elles ne remplissent pas les mêmes fonctions pour l'individu et la société et n'ont pas la même signification.

Seules des enquêtes intensives sur de petits échantillons, peut-être aussi des études à caractère anthropologique sur les cérémonies qui président à la formation des unions lou en de nombreux cas sur l'absence de cérémonie, pour ce qui est de l'union libre) nous en apprendront davantage sur ces aspectis. Yanagisako (1979) a cerné un jour en quelques mots les points de vue à explorer dans l'étude des familles et des ménages: composition ou structure; fonction; signification. Reconnaissons que l'analyse démographique de la formation et de la dissolution des unions au Canada a accompli un beau travail sur le premier point, et que cette tâche toujours indispensable se poursuit par les méthodes de l'analyse démographique traditionnelle, bien adaptées à ce propos.

Pour mieux comprendre le couple humain et ses comportements sexuels et reproductifs, il reste à élargir et à perfectionner l'analyse démographique, au moyen de données et de démarches d'analyse plus aptes à cerner les aspects qui ressortissent à la fonction et au sens. Dans la mesure où elle relèvera ce défi, la démographie du XXIe siècle sera plus solide comme science et plus utile à la décision.

\section{RÉFÉRENCES BIBLIOGRAPHIQUES}

ABBOTT, Andrew. 1983. "Sequences of social events: Concepts and methods for the analysis of order in social processes ", Historical Methods, 16, $4: 129-147$.

ABBOTT, Andrew. 1988. "Transcending general linear reality ", Sociological Theory, $6: 169-186$.

ABBOTT, Andrew. 1995. "Sequence analysis: New methods for old ideas ", Annual Review of Sociology, 21 : 93-113. 
BENOÎT, D., P. LÉVI et P. VIMARD. 1983. "Structures des ménages dans les populations rurales du Sud Togo ", Cahiers Orstom, Série sciences humaines, 19, 3.

BILLARI, Francesco, et Raffaella PICCARRETA. 1999. Studying Demographic Life Courses as Sequences. Communication présentée au séminaire international "Synthetic Biographies: State of the Art and Developments ", San Miniato, Italie, 6-9 juin, 30 p.

BLALOCK, H. M. 1960. Social Statistics. New York, McGraw-Hill.

BOURBEAU, Robert, et Valérie COURVILLE. 1997. "La mortalité violente selon l'âge, le sexe et la cause : un essai de classification des pays industrialisés, 1985-1989 ", European Journal of Population, $13: 71-94$.

BRACHER, Michael, Gigi SANTOW, S. Philip MORGAN et James TRUSSELL. 1993. "Marriage dissolution in Australia: Models and explanations ", Population Studies, $47: 403-426$.

BURCH, Thomas K. 1985. Family History Survey: Preliminary Findings. Ottawa, Statistique Canada, no 99-955 au catalogue.

BURCH, Thomas K. 1991. "De gustibus confusi sumus?", dans Jacques J. SIEGERS, Jenny DE JONG-GIERVELD et Evert VAN IMHOFF, éd. Female Labour Market Behaviour and Fertility: A Rational Choice Approach. Berlin, Springer-Verlag : 61-73.

CALDWELL, John C. 1982. Theory of Fertility Decline. New York, Academic Press, $386 \mathrm{p}$.

CALDWELL, John C., P. H. REDDY et Pat CALDWELL. 1988. The Causes of Demographic Change: Experimental Research in South India. Madison, University of Wisconsin Press, $285 \mathrm{p}$.

CASTRO MARTIN, Teresa. 1997. "Marriages without papers in Latin America ", International Population Conference, Beijing 1997. Liège, IUSSP, $2:$ 941-960.

CARMICHAEL, Gordon A. 1995. "Consensual partnering in the more developed countries", Joumal of the Australian Population Association, $12: 51-86$.

CERBARA, Loredana. 1997. "New statistical methods in population studies ", Demotrends, 1:6, Rome, Istituto di Ricerche sulla Popolazione.

COLEMAN, J. S. 1964. Introduction to Mathematical Sociology. New York, Free Press.

DENZIN, Norman K., et Yvonna S. LINCOLN. 1998. "Introduction: Entering the field of qualitative research ", dans Norman K. DENZIN et Yvonna S. LINCOLN, éd. Collecting and Interpreting Qualitative Materials. Thousand Oaks, Sage Publications : 1-34.

DE ROSE, Alessandra. 1992. "Socio-economic factors and family size as determinants of marital dissolution in Italy ", European Sociological Review, $8: 71-91$. 
FRICKE, Tom. 1997. "The uses of culture in demographic research: A continuing place for community studies ", Population and Development Review, 23, 4 : 825-832.

GREENHALGH, Susan. 1996. "The social construction of population science: An intellectual, institutional and political history of twentieth-century demography ", Comparative Studies in Society and History, 38, $1: 26-66$.

GREENHALGH, Susan. 1997. "Methods and meanings: Reflections or disciplinary differences ", Population and Development Review, 23, $4: 819-824$.

HALL, David. 1992. Reproductive Individualism and Divorce: An Examination of Attitudes and Marriage Dissolution in Canada. University of Western Ontario, thèse de Ph.D.

HAKKERT, R. 1992. Computing in Demographic Analysis: Beyond Paper and Pencil Algorithms. Communication présentée au IUSSP/ NIDI Expert Meeting on Demographic Software and Computing, La Haye, 29 juin-3 juillet.

HENRY, Louis, 1972. Démographie. Analyses et modèles. Paris, INED, $338 \mathrm{p}$.

KERTZER, David I. 1993. Sacrificed for Honor: Italian Infant Abandonment and the Politics of Reproductive Control. Boston, Beacon Press.

KERTZER, David I., et Tom FRICKE. 1997. "Toward an anthropological demography ", dans David I. KERTZER et Tom FRICKE, éd. Anthropological Demography: Towards a New Synthesis. Chicago, University of Chicago Press : 1-35.

KNODEL John, 1997. "A case for non-anthropological qualitative methods for demographers ", Population Development Review, 23, 4 : 847-853.

KRAVDAL, Øystein. 1997. “Wanting a child without a firm commitment to the partner: Interpretations and implications of a common behaviour pattern among Norwegian cohabitants ", European Journal of Population, $13: 269-298$.

LIAO, Futing. 1989. "Estimating fuzzy set membership coefficients with log-multiplicative association models: The case of contraceptiveness ", Mathematical Population Studies, 1 : 357-376.

LILLARD, Lee A., Michael J. BRIEN et Linda J. WAITE. 1995. "Premarital cohabitation and subsequent marital dissolution: A matter of self-selection? ", Demography, $32: 437-457$.

MCNICOLL, Geoffrey. 1992. "The agenda of population studies: A commentary and complaint ", Population and Deveiopment Review, 18 : 399-420.

PODHISTA, Chai, et al. 1990. "Women's work and family size in rural Thailand ", Asia-Pacific Population Journal, 5, 2 : 31-52.

PRESTON, Samuel. 1993. "The contours of demography: Estimates and projections ", Demography, 30, 4 : 593-606. 
RAVANERA, Zenaida, Fernando RAJULTON et Thomas K. BURCH. 1998. "Early life transitions of Canadian women: A cohort analysis of timing, sequences, and variations ", European Journal of Population, 14 : 179-204.

RICHARDS, Thomas, et Lyn RICHARDS. 1998. "Using computers in qualitative research ", dans Norman K. DENZIN et Yvonna S. LINCOLN, éd. Collecting and Interpreting Qualitative Materials. Thousand Oaks, Sage Publications : 211-245.

RINDFUSS, Ronald R., et Audrey VANDEN HEUVEL. 1990. "Cohabitation: A precursor to marriage or an alternative to being single? ", Population and Development Review, 16 : 703-726.

ROUSSEL, Louis. 1984. "Du pluralisme des modèles familiaux dans les sociêtés post-industrielles: quelques problèmes méthodologiques et théoriques ", dans Association internationale des démographes de langue française. Les Familles d'aujourd'hui. Colloque de Genève, septembre 1984, no 2 : 143-151.

SANTOW, Gigi, et Michael BRACHER. 1997. "Whither marriage? Trends, correlates, and interpretations ", International Population Conference, Beijing 1997. Liège, IUSSP, 2 : 919-939.

SABOURIN, Paul. 1993. "La régionalisation du social : une approche de l'étude de cas en sociologie ", Sociologie et sociétés, 25, 2 : 69-91.

STRAUSS, Anselm, et Juliet CORBIN. 1998. "Grounded theory methodology: An overview ", dans Norman K. DENZIN et Yvonna S. LINCOLN, éd. Strategies for Qualitative Inquiry. Thousand Oaks, Sage Publications : 158-183.

TESH, Renata. 1992. Qualitative Research. Analysis Types and Software Tools. New York, The Falmer Press, 330 p.

VILLENEUVE-GOKALP, Catherine. 1990. "Du mariage aux unions sans papier : histoire des transformations conjugales ", Population, $45,2: 265-298$.

VILLENEUVE-GOKALP, Catherine. 1997. "Vivre en couple chacun chez soi ", Population, 52, 5 : 1059-1081.

YANAGISAKO, S. J. 1979. "Families and households: The analysis of domestic groups ", Annual Review of Anthropology, 8 : 161-205. 